\title{
A state-independent network of depressive, negative and positive symptoms in male patients with schizophrenia spectrum disorders
}

\author{
Geeske van Rooijen ${ }^{\mathrm{a}, *}$, Adela-Maria Isvoranu ${ }^{\mathrm{b}}$, Olle H. Kruijt ${ }^{\mathrm{a}}$, Claudia D. van Borkulo ${ }^{\text {b,c }}$, Carin J. Meijer ${ }^{\text {, }}$ \\ Johanna T.W. Wigman ${ }^{\text {c }}$, Henricus G. Ruhé ${ }^{\text {a,d }}$, Lieuwe de Haan ${ }^{\text {a }}$, GROUP investigators:
}

Genetic Risk and Outcome of Psychosis investigators Richard Bruggeman ${ }^{2}$, Wiepke Cahn ${ }^{3}$, Lieuwe de Haan ${ }^{1}$, René S. Kahn ${ }^{3}$, Carin Meijer ${ }^{1}$, Inez Myin-Germeys ${ }^{6}$, Jim van Os ${ }^{4,5}$, Agna A. Bartels-Velthuis ${ }^{2}$

${ }^{1}$ University of Amsterdam, Academic Medical Center, Department of Psychiatry, Meibergdreef 5, 1105 AZ Amsterdam, The Netherlands

2 University of Groningen, University Medical Center Groningen, University Center for Psychiatry, Hanzeplein 1, 9700 RB, Groningen, The Netherlands

${ }^{3}$ Department of Psychiatry, Rudolf Magnus Institute of Neuroscience, University Medical Center Utrecht, Utrecht, The Netherlands ${ }^{1}$

${ }^{4}$ South Limburg Mental Health Research and Teaching Network, EURON, Maastricht University Medical Center, Maastricht, The Netherlands ${ }^{2}$

${ }^{5}$ King's College London, King's Health Partners, Department of Psychosis Studies, Institute of Psychiatry, London, United Kingdom

${ }^{6}$ KU Leuven, Department of Neuroscience, Research Group Psychiatry, Center for Contextual Psychiatry, Leuven, Belgium ${ }^{3}$

a University of Amsterdam, Academic Medical Center, Department of Psychiatry, Meibergdreef 5, 1105 AZ Amsterdam, The Netherlands

b University of Amsterdam, Department of Psychology, Psychological Methods, Nieuwe Achtergracht 129-B, 1018 WT Amsterdam, The Netherlands

c University of Groningen, University Medical Center Groningen, University Center for Psychiatry, Hanzeplein 1, 9700 RB Groningen, The Netherlands

${ }^{\mathrm{d}}$ Warneford Hospital, Department of Psychiatry, University of Oxford, United Kingdom

\section{A R T I C L E I N F O}

\section{Article history:}

Received 30 May 2017

Received in revised form 14 July 2017

Accepted 15 July 2017

Available online $\mathrm{xxxx}$

\section{Keywords:}

Depressive symptoms

Positive symptoms

Negative symptoms

Schizophrenia

Network comparison testNetwork analysis

\begin{abstract}
A B S T R A C T
Depressive symptoms occur frequently in patients with schizophrenia. Several factor analytical studies investigated the associations between positive, negative and depressive symptoms and reported difficulties differentiating between these symptom domains. Here, we argue that a network approach may offer insights into these associations, by exploring interrelations between symptoms. The aims of current study were to I) construct a network of positive, negative and depressive symptoms in male patients with schizophrenia to investigate interactions between individual symptoms; II) identify the most central symptoms within this network and III) examine group-level differences in network connectivity between remitted and non-remitted patients. We computed a network of depressive, positive and negative symptoms in a sample of 470 male patients diagnosed with a psychotic disorder. Depressive symptoms were assessed with the Calgary Depression Rating Scale for Schizophrenia, while psychotic symptoms were assessed with the Positive and Negative Syndrome Scale. Networks of male patients who fulfilled remission criteria (Andreasen et al., 2005) and non-remitters for psychosis were compared.

Our results indicate that depressive symptoms are mostly associated with suicidality and may act as moderator between psychotic symptoms and suicidality. In addition, 'depressed mood', 'observed depression', 'poor rapport', 'stereotyped thinking' and 'delusions' were central symptoms within the network. Finally, although remitted male patients had a similar network structure compared to non-remitters the networks differed significantly in terms of global strength. In conclusion, clinical symptoms of schizophrenia were linked in a stable way, independent of symptomatic remission while the number of connections appears to be dependent on remission status.
\end{abstract}

(c) 2017 Elsevier B.V. All rights reserved.

\footnotetext{
* Corresponding author.

E-mail addresses: g.vanrooijen@amc.uva.nl (G.van Rooijen), isvoranu.adela@gmail.com (A.-M. Isvoranu), o.h.kruijt@amc.uva.nl (O.H. Kruijt), cvborkulo@gmail.com (C.D.van Borkulo), c.j.meijer@amc.uva.nl (C.J. Meijer), j.t.w.wigman@umcg.nl (J.T.W.Wigman), ruhe@gmail.com (H.G. Ruhé), l.dehaan@amc.uva.nl (L. de Haan).

1 w.cahn@umcutrecht.nl; r.kahn@umcutrecht.nl.

2 j.vanos@maastrichtuniversity.nl.

3 inez.germeys@kuleuven.be.
} 


\section{Introduction}

Patients with schizophrenia may present with a wide variety of symptoms: positive and negative symptoms are considered core features of schizophrenia, but depressive symptoms are also common, with a modal prevalence rate of 25\% (Buckley et al., 2009; Siris, 2000). In order to study the associations between symptoms, a network approach might be advantageous - in comparison to traditional factor-analytic approaches, network models offer the possibility to study potential interactions between individual symptoms (Borsboom, 2017; Borsboom and Cramer, 2013). Specifically, within a network perspective, it is presumed that mental health problems result from complex interactions between individual symptoms, which influence and reinforce each other, instead of originating from an underlying latent disorder (Borsboom and Cramer, 2013).

In the past years the network approach has been increasingly applied to study psychopathology ((Isvoranu et al., 2016; Isvoranu et al., 2017; van Rooijen et al., 2017); for a review see (Fried et al., 2016)). For instance, Wigman and colleagues (2015) showed that the networks of individuals with a psychiatric diagnosis contained positive feedback loops, which may explain a 'downward spiral of negative mental states', which are clinically recognizable in the way symptoms of psychosis can enhance each other. Isvoranu et al. (2017) have moved beyond symptom-symptom associations, integrating environmental risk factors into network models; they found that childhood trauma was associated with symptoms of general psychopathology and not directly to positive or negative symptoms. The network approach is therefore not bound to 'traditional' diagnostic categories - psychopathology is conceptualized as a complex system and the 'overlap' between symptoms and risk factors of different disorders is a source of valuable information rather than a problem to overcome.

Notably, a recent network paper using the baseline symptoms of the 'Genetic Risk and Outcome of Psychosis' (GROUP) study (KorverNieberg et al., 2012) showed that in male patients with schizophrenia the symptoms assessed by the Comprehensive Assessment of Symptoms and History (CASH; (Andreasen et al., 1992)) displayed strong within-and between- cluster interactions and formed a network with central symptoms such as 'loss of interest', 'chaotic speech', 'inability to enjoy recreational interest in activities', 'inability to form or maintain relationships with friends' and 'poverty of content of speech' (van Rooijen et al., 2017). Central symptoms have been argued to be relevant as targets for treatment interventions, as these symptoms are most likely to influence the other symptoms in the network. In addition, relations between suicidality, depressive and positive symptoms were investigated and based on the strong associations between depressive symptoms and suicidality and between delusional and depressive symptoms, but in the absence of a direct relationship between delusional symptoms and suicidality, it was hypothesized that delusional symptoms may activate depressive symptoms and influence suicidal thoughts via this pathway.

However, the CASH is limited in addressing current depressive symptoms, since within the CASH the DSM-IV criteria are investigated; these are known to show overlap with other symptoms in patients with schizophrenia (i.e., negative and extrapyramidal side effects; (Siris, 2000)). We therefore aimed to expand on the previous study and investigate the association between positive, negative and depressive symptoms further by constructing a network model that includes the Calgary Depression Rating Scale for Schizophrenia (CDSS; (Addington et al., 1990)), which is a validated instrument for assessing depression in patients diagnosed with schizophrenia (Lako et al., 2012). The CDSS was administered at first follow-up. We combined data from the CDSS and the Positive and Negative Syndrome Scale (PANSS; (Kay et al., 1987)) assessed at follow-up. In addition, the latter questionnaire was used to assess psychotic remission status. A previous study in depression showed that different severity symptom networks in depressed patients (at baseline) were associated with varying illness courses (van
Borkulo et al., 2015). In order to apply this type of profiling, first the stability (i.e., state-independence) of a network structure is required. However, this has not been investigated in patients with schizophrenia and was therefore the secondary aim of this study.

In summary, network analysis has been shown to help disentangle the interactions between individual symptoms of a disorder and as such we have employed this methodology in the current study in order to investigate the association between psychosis and depressive symptoms. The aims of current study were as follows: I) to construct a network of symptoms in male patients with a schizophrenia spectrum disorder in order to investigate how negative, positive and depressive symptoms interact, by using a validated questionnaires to asses depressive symptoms; II) to identify the most central symptoms within this network and III) to examine potential group-level differences in network connectivity between remitted and non-remitted patients. This might reveal important profiling information for prognosis.

\section{Methods}

\subsection{Subjects}

Data was collected as part of the longitudinal multicentre GROUP study, described in detail elsewhere (Korver-Nieberg et al., 2012). Here we used data from a GROUP subsample, consisting of male patients with non-affective psychotic disorders, diagnosed according to the Diagnostic and Statistical Manual of Mental Disorders (DSM-IV; (American Psychiatric Association, 2000)). Of note, we chose to include only male patients, given the known gender differences in symptomatology and the relatively small number of included female patients in GROUP (Hill, 2016; Morgan et al., 2008). Measurements of the GROUP study were collected at baseline, at 3 and 6-year follow-up. Because the CDSS was obtained in a large subsample at 3-year follow-up, we used data from this wave only.

\subsection{Symptom assessment}

The CDSS (Addington et al., 1990) was used to assess depressive symptoms. The CDSS is a nine-item structured interview, in which every item is rated on a scale ranging from 0 (absent) to 3 (severe) (Supplementary Table S1). The PANSS (Kay et al., 1987) was used to measure the severity of positive and negative symptoms. The PANSS consists of 30 items (Supplementary Table S1) in which each item is scored on a scale ranging from 1 (absent) to 7 (extreme) and it is divided into three subscales: positive, negative and general psychopathology (e.g., depression, anxiety and somatic concern) symptoms. The general psychopathology subscale was not included in our network, since inclusion of this subscale would have created a substantial overlap between with the items of the CDSS. In addition, we used the Andreasen et al. remission criteria (Andreasen et al., 2005) to assess whether a patient was in symptomatic remission at the time of assessment (i.e., during the second assessment of the GROUP-cohort). The Andreasen criteria constitute a symptom severity and a time criterion. The symptom severity criterion was determined by a score of 3 or lower on all of the following items: P1 (delusions), P2 (disorganization), P3 (hallucinatory behavior), G5 (mannerisms/posturing), G9 (unusual thought content), N1 (blunted affect), N4 (passive social withdrawal), and N6 (lack of spontaneity). For the time criterion we assessed whether a symptomatic remission had been maintained for 6 months or longer prior to the time of assessment (i.e., 6 months before the assessment).

\subsection{Statistical analysis}

\subsubsection{Network construction}

We constructed a symptom network as previously described (Borsboom and Cramer, 2013; Epskamp et al., 2017; van Rooijen et al., 2017) of positive, negative and depressive symptoms. In the generated 
network model, individual items (i.e., symptoms) were represented as nodes and associations between them as edges.

A non-paranormal transformation (i.e., a tool for relaxing the normality assumption) was performed prior to the analysis, since current data were not normally distributed (Liu et al., 2009). For constructing the generated network, the $R$ package qgraph was used ((Epskamp et al., 2012); R (Core Team, 2016)). The network structure was based on L1-regularized partial correlations (Friedman et al., 2008; Tibshirani, 1996). For estimating the relations between symptoms, partial correlations are chosen over zero-order correlation (i.e., correlation between two variables), since zero-order correlations can be spurious (i.e., resulting from indirect interactions). Moreover, L1-regularization guarantees an optimal balance between parsimony and goodness of fit of the network model. A specific form of L1-regularization, LASSO regularization, encompasses model selection with the Extended Bayesian Information Criterion (EBIC), which uses a so-called hyperparameter $\gamma$ (Chen and Chen, 2008; Foygel and Drton, 2015; Foygel and Drton, 2010; van Borkulo et al., 2014). The details of the influence of $\gamma$ on the network have been published earlier (van Borkulo et al., 2015). In the generated network the hyperparameter was set to 0.5 , which showed an optimal balance between a network with many connections ( $\gamma=$ 0 ) and a network with minimal connections $(\gamma=1)$. The layout used when computing the networks was derived from the FruchtermanReingold algorithm, which computes the optimal layout so that nodes with stronger and/or more connections are placed closer and more central to each other (Fruchterman and Reingold, 1991). We primarily displayed the correlation results in a figure as the applied Fruchterman-Reingold algorithm simultaneously shows the strength of associations between different symptoms (thickness of the connections) as well as the direction of these associations (i.e., positive or negative association) in the network.

\subsubsection{Network analysis}

Based on earlier network studies, we expected that some symptoms would cluster strongly. Therefore, in our results we will use the term 'communities' (i.e., a part of the total network that contains strongly connected items). At first, a symptom network was constructed for the total sample of male subjects. Communities and important symptom interactions were described, with a specific focus on the relations between delusional and depressive symptoms (including suicidality). Moreover, we analysed the importance of each node by investigating the following centrality measures: 'node strength', 'closeness' and 'betweenness' (Barrat et al., 2004; Boccaletti et al., 2006; Opsahl et al., 2010). For a description on centrality measures see Supplementary Methods. In the second step of the analyses, the symptom network of male patients who were in remission (based on the PANSS-remission tool) was compared to the network of male patients who were not in remission. In order to compare networks of remitted and non-remitted male patients, we used a network comparison test (NCT; (van Borkulo et al., 2017)), which is a permutation test (1000 iterations) in which the difference between networks of two groups (i.e., remitters and non-remitters) is calculated repeatedly for randomly regrouped individuals. The NCT is implemented in the $R$ package 'NetworkComparisonTest'( $\mathrm{R}$ (Core Team, 2016; van Borkulo, 2016)). By using the NCT it is possible to compare two (independent) networks based on i) network structure and ii) overall global strength. For details on the NCT see Supplementary Methods.

\subsubsection{Additional analyses}

We performed a stability check to investigate the stability of the generated networks (Epskamp et al., 2017). Moreover we used Exploratory Graph Analyses (EGA) to detect highly connected clusters of symptoms (i.e., communities; (Golino and Epskamp, 2016)). For a detailed description of both analyses see the Supplementary Methods.

\section{Results}

\subsection{Study sample}

After removing missing data, 470 male patients were included in the analyses (from which $32 \%$ were in remission; see Table 1 ). Mean and median scores of positive, negative and depressive symptoms are presented in Supplementary Table S1. Moreover, the derived correlation matrix between the different items is presented in Supplementary Table S2. The (overall) mean score of the CDSS was 1.07 (SD = 2.04) for patients in symptomatic remission from psychosis and 2.5 (SD = 3.1) for patients not in remission, respectively. A commonly used cutoff value of the CDSS of $\geq 6$ was used (Sarró et al., 2004), $17.1 \%$ of the patients in the psychotic group suffered from depression. In the non-psychotic (remission) group this was 5.3\% (Table 1). The time criterion of the psychotic remission criteria was lacking in 4 patients (of whom 3 were in symptomatic remission). We therefore excluded these patients from the comparisons between remission and non-remission.

\subsection{Network including all patients (Fig. 1)}

The network of negative, positive and depressive symptoms is presented in Figure 1; symptoms from the original subscales (depressive, positive and negative symptoms) are shown in different colours. In general, all symptoms within the network were connected. Of note, the stability check showed considerable overlap between bootstrapped confidence intervals (CIs), indicating that the generated network should be interpreted with caution regarding the differences between edgeweights (see Supplementary Material Figs. S1-S3). Of note, fit indices (e.g., RMSEA/BIC indices) to contrast for parsimony can be extracted from the qgraph package 'ggmFit'. However, there are currently several problems with such fit indices in network models and this method has not been validated. Consequently, we decided to not report these fit indices.

\subsubsection{Communities}

We used EGA to identify 3 highly connected clusters of symptoms (i.e., communities) within the original three subscales (i.e., positive and negative subscales of the PANSS and the depressive symptoms as derived from the CDSS; see Supplementary Fig. S4). The depressive symptoms formed 1 community; within this community strong connections between D2 (hopelessness) and D8 (suicide), as well as between D9 (observed depression) and D1 (depressed mood) were prominent. The second community was formed of all positive symptoms, including

Table 1

Demographics and clinical characteristics ${ }^{\mathrm{c}}$.

\begin{tabular}{|c|c|c|c|}
\hline & $\begin{array}{l}\text { Male } \\
\text { participants } \\
(n=470)\end{array}$ & $\begin{array}{l}\text { Males } \\
\text { remission } \\
(n=150)\end{array}$ & $\begin{array}{l}\text { Males non - } \\
\text { remission }^{\mathrm{a}} \\
(n=316)\end{array}$ \\
\hline Age at inclusion, years (mean \pm SD) & $26.9(6.6)$ & $25.99(6.60)$ & $27.2(6.59)$ \\
\hline Number of episodes (mean \pm SD) & $2.21(1.41)$ & $1.94(1.33)$ & $2.36(1.44)$ \\
\hline CDSS total (mean $\pm S D$ ) & $2.1 \pm 2.9$ & $1.07(2.04)^{\mathrm{b}}$ & $2.5(3.1)^{\mathrm{b}}$ \\
\hline CDSS (\%), no depression (score $\leq 5$ ) & $407(86.6)$ & $142(94.7)^{\mathrm{b}}$ & $262(82.91)^{\mathrm{b}}$ \\
\hline \multicolumn{4}{|l|}{ PANSS (Mean, SD) } \\
\hline Positive symptoms & $1.68(0.70)$ & $1.87(0.75)$ & $1.27(0.31)$ \\
\hline Negative symptoms & $1.75(0.76)$ & $1.93(0.83)$ & $1.37(0.39)$ \\
\hline \multicolumn{4}{|l|}{ Diagnosis } \\
\hline Schizophrenia (\%) & $371(78.9)$ & $110(73.33)$ & $259(81.96)$ \\
\hline Schizoaffective disorder (\%) & $83(17.7)$ & $27(18.0)$ & $54(17.09)$ \\
\hline Schizophreniform disorder (\%) & $16(3.4$ & $13(8.67)$ & $3(0.95)$ \\
\hline $\begin{array}{l}\text { Use of antipsychotic medication, } \\
\mathrm{n}(\% \text { yes })^{\mathrm{c}}\end{array}$ & $366(77.9)$ & $110(73.3)$ & $254(80.4)$ \\
\hline
\end{tabular}




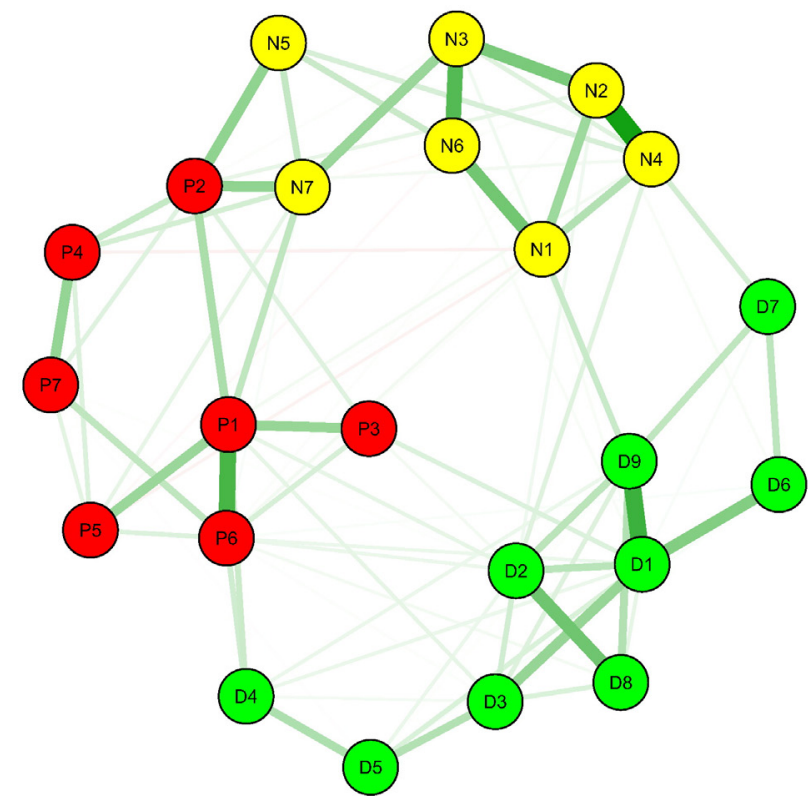

- Positive Symptoms

- Negative Symptoms

- Depressive Symptoms

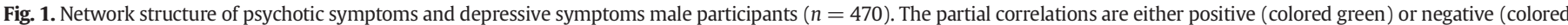

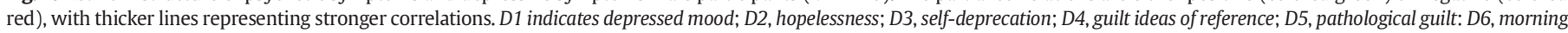

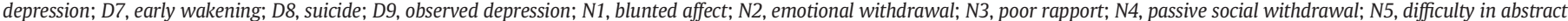

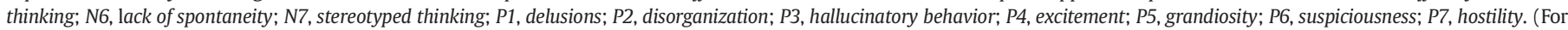
interpretation of the references to color in this figure legend, the reader is referred to the web version of this article.)

the negative symptoms N5 (difficulty in abstract thinking) and N7 (stereotyped thinking). Within this community, clustering between P1 (delusions) and P6 (suspiciousness), and P3 (hallucinatory behavior) and P5 (grandiosity) were the strongest. The last community was formed by the remaining negative symptoms: N1 (blunted affect), N2 (emotional withdrawal), N3 (poor rapport), N4 (passive social withdrawal) and N6 (lack of spontaneity).

\subsubsection{Interrelatedness between depressive and delusional symptoms}

There was one association between depressive and positive symptoms, namely between D8 (suicide) with P6 (suspiciousness). Of note, between suicide (D8) and delusions (P1) was no direct connection. However, P1 (delusions) was connected with (other) depressive symptoms, including D2 (hopelessness), D3 (self-deprecation) and D4 (guilt ideas of reference).

\subsection{Centrality measures}

D1 (depressed mood), D9 (observed depression), N3 (poor rapport), N7 (stereotyped thinking) and P1 (delusions) showed highest centrality measures (Fig. 2), indicating that these symptoms may be important symptoms within this network.

\subsection{Differences between remitters and non-remitters (Fig. $3 A+B)$}

The NCT analysis comparing the symptom network of male patients in remission of psychosis (based on the PANSS remission tool) versus the network of male patients not in remission of psychosis showed significant differences in global strength $(P=0.04)$, but not in terms of network structure $(P=0.39)$. The fact that the test about network structure invariance yielded no significant differences indicates that the null hypothesis cannot be rejected because there are no edges that differ more than can be expected. The fact that the overall global strength did differ significantly might be due to more and/or stronger edges. Combining both findings (i.e., no significant difference between network structure indicating no difference in edges, but significant differences regarding global strength), it is more plausible that the significant difference regarding the overall global strength is driven by more edges, rather than a few strong edges. Since there were no significant differences between the networks regarding network structure, we did not pursue with further testing of specific edges (i.e., further testing could lead to Type I errors) (van Borkulo et al., 2017). These results of the NCT may be explained by the fact that in the network of the remitted patients less edges were present compared to the network of the nonremitted patients (of note, this may be, in part, due to sample size differences). For example, a community (i.e., highly connected cluster of symptoms) was no longer present within the symptoms of the positive subscale of the remitters. The association between P1 (delusions) and other depressive symptoms disappeared in the network of the remitted patients. Interestingly, in the network of the remitted psychotic patients, no associations existed between symptoms originating from the positive and depressive subscales. This was also the seen between the negative and depressive subscales.

\section{Discussion}

This study investigated the interrelatedness of positive, negative and depressive symptoms in male patients with non-affective psychosis, using a network approach. As a first main finding, we identified the symptoms 'depressed mood', 'observed depression', 'poor rapport', 'stereotyped thinking', 'delusions' and 'suspiciousness' as being important central symptoms with strong associations with other symptoms. The second main finding is that the networks of remitters and non-remitters are comparable regarding network structure, although the networks differed regarding overall global strength. Symptoms with high centrality measures may be important symptoms as potential targets for treatment interventions, while the differences in global strength indicate that the number of connections between symptoms seems to be dependent on illness state. Furthermore, we identified important interactions between individual symptoms, for example, between depressive symptoms and delusions. Based on these associations we hypothesize that depressive symptoms might be able to activate suicidality, while positive symptoms may trigger depressive symptoms and hereby influencing suicidality. This interrelatedness of symptoms may further increase our understanding of psychopathology and provides important information for profiling. 

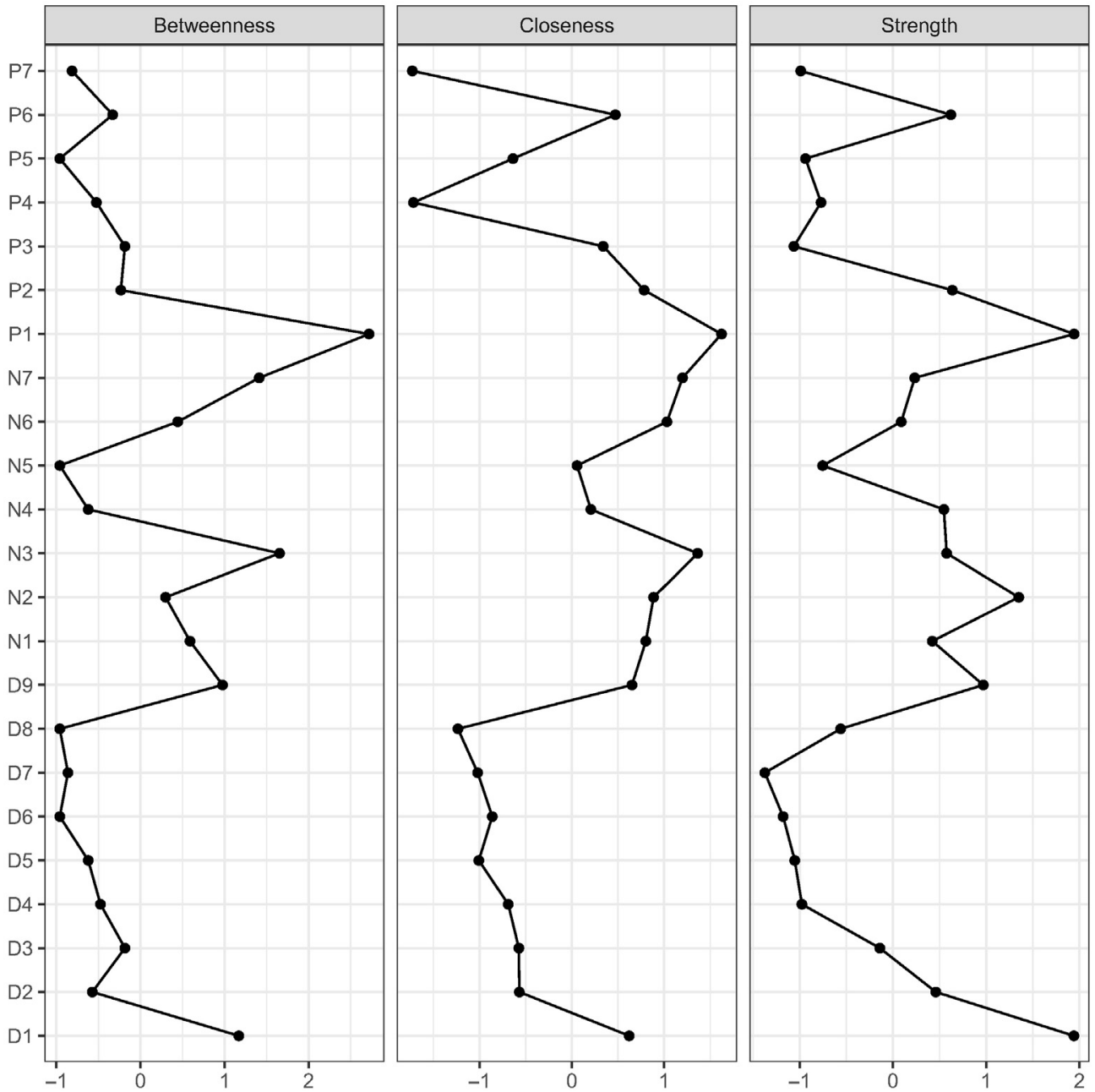

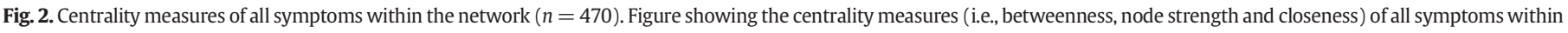

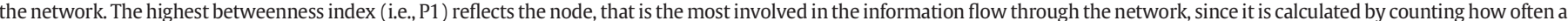

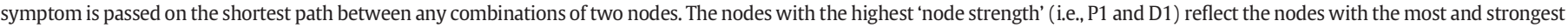

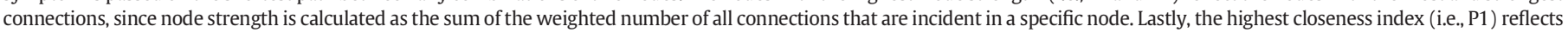
the node that is most easy to reach, since it is calculated as the inverse of the mean shortest distance to all other nodes. For the abbreviations of the items, see Fig. 1.

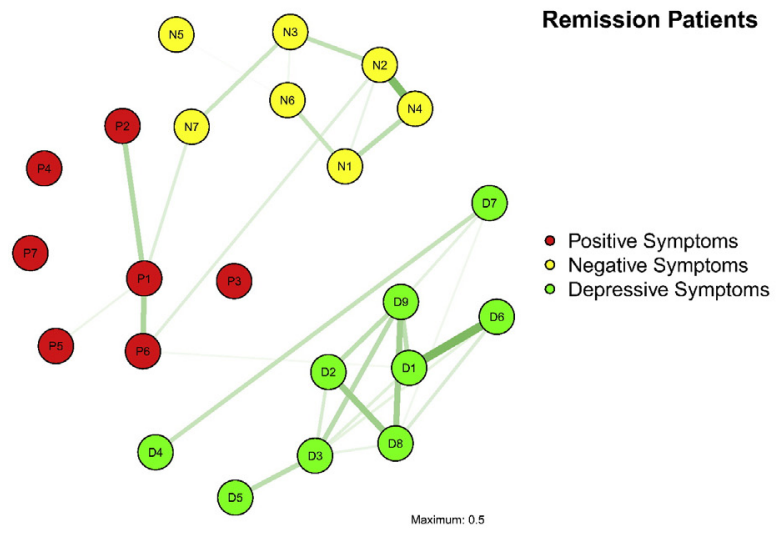

A.

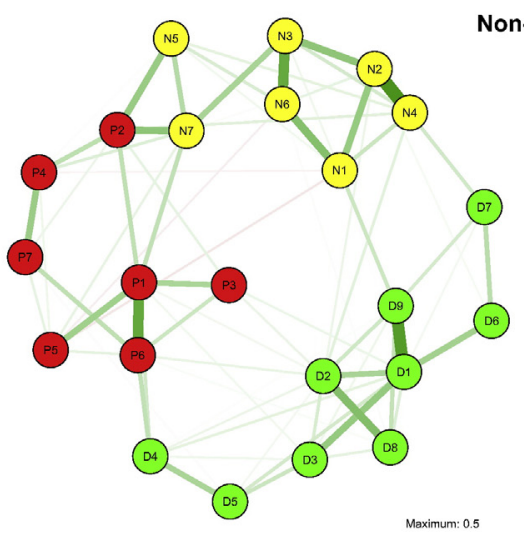

Non-Remission Patients
- Positive Symptoms

- Depressive Symptoms

B.

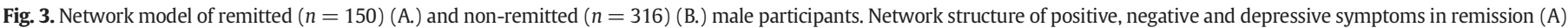

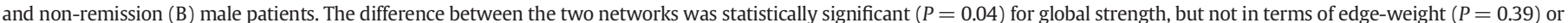

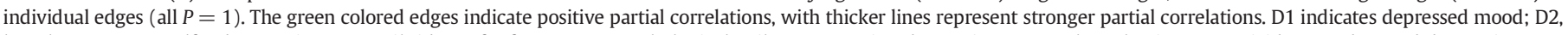

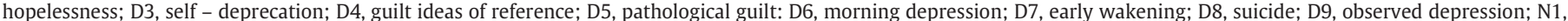

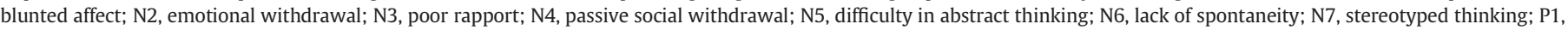

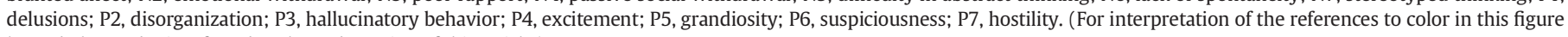
legend, the reader is referred to the web version of this article.) 


\subsection{Symptoms networks and remission of psychotic symptoms}

Remission status of psychosis influenced only the overall global strength between symptoms; the remitted patients showed a network that was not significantly different in structure, but had fewer connections than the network in non-remitted psychotic patients. To the best of our knowledge, no earlier study investigated the influence of state on networks in patients with non-affective psychosis. Of note, our results are based on group-level data and it is important to acknowledge that group-level results may not be generalizable to individual networks (earlier discussed by (Bos and Wanders, 2016; van Borkulo et al., 2016)). It is currently unknown to what degree networks of an individual match the network at group level (Fried et al., 2016).

If we assume that our group-level results are representative for individuals, our finding that non-remitted patients showed a stronger connected network supports the hysteresis principal of the network theory (Borsboom, 2017). This idea posits that mental disorders should be interpreted as complex dynamical systems in which symptoms are able to influence each other, ultimately creating self-reinforcing feedback loops. The hysteresis principal implies that the self-reinforcing nature of symptom activation is more likely to take place in more strongly connected networks (i.e. networks with more and/or stronger edges; (Borsboom, 2017)). Therefore, the observed difference in global strength but not in structure between the remitted psychosis and non-remitted psychosis, may be explained by the presence of a more strongly connected network during an active psychosis, possibly due to self-reinforcing loops of symptom activation, which might play an important role in the maintenance of psychopathology.

Interestingly, both groups suffered from depressive symptoms (although the remitted patients showed significantly less depression i.e., $5.3 \%$ versus $17.1 \%$ respectively). In the network of the remitted psychotic group no associations existed between depressive symptoms and symptoms of the other subscales, while in the non-remitted group several edges connected the depressive symptoms with symptoms of the other subscales. This suggests that especially in the non-remitted group symptoms of different subscales co-occur and might activate each other. However, future longitudinal studies are required to investigate how symptom networks change, first to a different state (e.g. from absence of symptoms towards manifest psychosis or vice versa), and second in relation to external stimuli (e.g., after stressful events and/ or discontinuation of treatment) and third whether patterns of network connectivity are also related to the course of illness, as shown in depressed patients (van Borkulo et al., 2015). This is also important to pursue given the differences in sample size between our two groups, which make it difficult to disentangle whether edge absence is dependent on the sample size or remission criterion.

\subsection{Centrality measures}

The symptoms 'depressed mood', 'observed depression', 'poor rapport', 'stereotyped thinking' and 'delusions' showed to have high centrality within our network. This implies that these symptoms might be relevant as targets for treatment interventions, as these symptoms are most likely to influence several other symptoms. Although comparing between different network studies is challenging, due to the use of different questionnaires measuring different constructs of symptoms, current results are in concordance with results from previous studies (Fried et al., 2016; Levine and Leucht, 2016; Madhoo and Levine, 2016; van Rooijen et al., 2017). In our earlier performed network analysis in the same sample using different questionnaires and at a different time point, central symptoms included, among others, items reflecting the social participation of patients (i.e., loss of interest, inability to enjoy recreational activities and inability to maintain relationship with friends; (van Rooijen et al., 2017)). In the current study, centrality of the symptom 'poor rapport' underlines the importance of symptoms reflecting the social participation of patients. Although measured by a different questionnaire, Levine and Leucht (Levine and Leucht, 2016) constructed a network of only negative symptoms and showed in their baseline network that 'poverty of content speech' had, among others, one of the highest closeness indexes from which there is overlap with the central symptom 'stereotyped thinking' in current study. Additionally, in depressed patients the most frequently reported centrality symptoms are 'depressed and loss of interest/pleasure' and 'energy/fatigue' (Fried et al., 2016; Madhoo and Levine, 2016), from which the first symptom is also in line with our results, suggesting that depressive symptoms have an important role in maintaining symptoms across different psychiatric disorders; these may be important transdiagnostic targets for treatment interventions across different psychiatric disorders. Future research should elaborate on these findings by investigating whether targeting these central symptoms might indeed lead to better outcomes, as the importance of central symptoms is currently only theoretically based (Wichers et al., 2017).

\subsection{The relations between suicide, depressive and delusional symptoms}

As highlighted above, in our previous network study in the same sample but with different symptom scales and a different point of time (van Rooijen et al., 2017) we found no direct connection between delusional symptoms and 'recurrent thoughts of suicide' but instead several connections between delusional and depressive symptoms. Based on these findings we suggested that delusional symptoms seem to activate depressive symptoms and via this pathway influenced suicidal thoughts. In the current study, which including a validated questionnaire to measure depressive symptoms in psychotic-patients, we found similar results since 'suicide' was connected to several depressive symptoms, however, only one association was present with the positive symptom 'suspiciousness'. Moreover, the symptom 'delusions' was connected with several (other) depressive symptoms. This finding is also in line with an earlier study, where Bornheimer (Bornheimer, 2016) showed that depressive symptoms were moderated by positive symptoms in predicting suicidal ideation. Thus, it could be hypothesized that depressive symptoms are linked to suicidality, while positive symptoms especially trigger depressive symptoms and hereby influence suicidality. Of note, this hypothesis is based on the assumption that our resulting network displays potential causal relationships and that it is representative for the network structure within individual patients.

\subsection{Limitations}

As the network approach is developing there are several issues of debate. Some limitations are also applicable to current study; firstly, results of the performed stability check indicated that the generated network should be interpreted with caution due to the overlapping confidence intervals, especially when investigating differences in edgeweights of the network. As a result further studies with larger datasets are needed to replicate our findings. Secondly, as discussed in-depth in comments on earlier published network studies we should be careful with generalizing results to individuals since the generated networks are based on group-level analysis (Bos and Wanders, 2016; Fried et al., 2016). This is especially important in the interpretation of centrality measures for treatment interventions: ideally, within-person network should be investigating to help determine the central symptoms within these networks; this may help guide personalized treatment interventions. Thirdly, the naturalistic study design does not allow a control for the effect of differences in current treatment; for example the differences in overall global strength between remitters and non-remitters could be due to differences in medication use or compliance. Fourthly, it could be hypothesized that the difference in global strength is a result of reduced severity of symptoms in those who are in remission, represented by decreased mean sum-scores of positive, negative and to a lesser extent depressive symptoms in remitted patients. However, van 
Borkulo et al. (van Borkulo et al., 2015) argued that the level of mean scores does not necessarily influence the generated network. Likewise, a lower score of items does not automatically lead to weaker associations between these items. Nevertheless, factors that are related to severity, such as variance in symptom scores (i.e., due to a floor/ceiling effect) in one of the groups might still lead to different levels of network connectivity. A fifth limitation is that due to a low number of female participants, there was insufficient data to perform separate analyses in female participants and compare male-female networks. Given known differences between men and women in terms of onset, course and nature of psychotic symptomatology (Leung and Chue, 2000), extrapolation of our results to women should be done with caution. Lastly, based on the cross-sectional design of our study we are unable to establish causality, which makes our conclusions regarding interactions between individual symptoms hypothesis driven. In line with this limitation, remission status was assessed at 3-years of follow-up after baseline and the 6-months time criterion was assessed retrospectively. Consequently, a recall bias could have taken place with patients having difficulties with remembering symptom severity up to 6 months prior to assessment. Moreover, the items G5 (mannerisms/posturing) and G9 (unusual thought content) are both part of the Andreasen et al. remission criteria (Andreasen et al., 2005), however, we did not include all symptoms of the general psychopathology scale in our network analyses. Given the wide application of the Andreasen et al. criteria (Andreasen et al., 2005) in current literature we chose to apply the original and complete criteria.

In conclusion, in the current study we constructed a network to highlight interrelations between psychotic and depressive symptoms and identified symptoms with high centrality measures, indicating that these symptoms are important within the network and might be potential targets for treatment interventions. In addition, we replicated in part that depressive symptoms may moderate the relation between psychotic symptoms and suicidality, and in addition we showed that the number of connections between symptoms differed between remitted versus non-remitted psychotic male patients. These findings on symptom level may be informative to generate hypotheses regarding the maintenance and development of psychopathology.

\section{Contributors}

Geeske van Rooijen (GvR) and Carin Meijer (CM) performed the literature search which formed the basis of current study. GvR, Adela-Maria Isvoranu (AI) and Claudia D. van Borkulo undertook the network analysis. The first draft of the manuscript was written by GvR. AI, CM, Olle H. Kruijt, Johanna T.W. Wigman, Henricus G. Ruhé, Lieuwe de Haan revised the manuscript more than once. All authors, including the GROUP investigators, approved the manuscript in its current form.

\section{Funding}

The infrastructure for the GROUP study is funded through the Geestkracht programme of the Dutch Health Research Council (Zon-Mw, grant number 10-000-1001), and matching funds from participating pharmaceutical companies (Lundbeck, AstraZeneca, Eli Lilly, Janssen Cilag) and universities and mental health care organizations (Amsterdam: Academic Psychiatric Centre of the Academic Medical Center and the mental health institutions: GGZ Ingeest, Arkin, Dijk en Duin, GGZ Rivierduinen, Erasmus Medical Centre, GGZ Noord Holland Noord. Groningen: University Medical Center Groningen and the mental health institutions: Lentis, GGZ Friesland, GGZ Drenthe, Dimence, Mediant, GGNet Warnsveld, Yulius Dordrecht and Parnassia psycho-medical center The Hague. Maastricht: Maastricht University Medical Centre and the mental health institutions: GGZ Eindhoven en De Kempen, GGZ Breburg, GGZ Oost-Brabant, Vincent van Gogh voor Geestelijke Gezondheid, Mondriaan, Virenze riagg, Zuyderland GGZ, MET ggz, Universitair Centrum Sint-Jozef Kortenberg, CAPRI University of Antwerp, PC Ziekeren Sint-Truiden, PZ Sancta Maria Sint-Truiden, GGZ Overpelt, OPZ Rekem. Utrecht: University Medical Center Utrecht and the mental health institutions Altrecht, GGZ Centraal and Delta).

JTWW is supported by NWO VENI grant (no 016.156.019). HGR is supported by a ZonMW/NWO VENI grant (no 016.126.059).

\section{Conflict of interest}

None to declare.

\section{Acknowledgement}

We are grateful for the generosity of time and effort by the patients, their families and healthy subjects. Furthermore we would like to thank all research personnel involved in the GROUP project, in particular: Joyce van Baaren, Erwin Veermans, Ger Driessen, Truda Driesen, Karin Pos, Erna van't Hag, Jessica de Nijs, Atiqul Islam, Wendy Beuken and Debora Op't Eijnde.

\section{Appendix A. Supplementary data}

Supplementary data to this article can be found online at http://dx. doi.org/10.1016/j.schres.2017.07.035.

\section{References}

Addington, D., Addington, J., Schissel, B., 1990. A depression rating scale for schizophrenics. Schizophr. Res. 3, 247-251.

American Psychiatric Association, 2000. Diagnostics and Statistical Manual of Mental Disorders (4th Ed., Text rev.). Washington, DC.

Andreasen, N.C., Flaum, M., Arndt, S., 1992. The comprehensive sssessment of symptoms and history (CASH). An instrument for assessing diagnosis and psychopathology. Arch. Gen. Psychiatry 49, 615-623.

Andreasen, N.C., Carpenter, W.T., Kane, J.M., Lasser, R.A., Marder, S.R., Weinberger, D.R., 2005. Remission in schizophrenia: proposed criteria and rationale for consensus. Am. J. Psychiatry 162:441-449. http://dx.doi.org/10.1176/appi.ajp.162.3.441.

Barrat, A., Barthélemy, M., Pastor-Satorras, R., Vespignani, A., 2004. The architecture of complex weighted networks. Proc. Natl. Acad. Sci. U. S. A. 101:3747-3752. http:// dx.doi.org/10.1073/pnas.0400087101.

Boccaletti, S., Latora, V., Moreno, Y., Chavez, M., Hwang, D.U., 2006. Complex networks: structure and dynamics. Phys. Rep. 424:175-308. http://dx.doi.org/10.1016/ j.physrep.2005.10.009.

van Borkulo, C., 2016. Package NetworkComparionTest. (https://cran.r-project.org).

van Borkulo, C.D., Borsboom, D., Epskamp, S., Blanken, T.F., Boschloo, L., Schoevers, R.A., Waldorp, L.J., 2014. A new method for constructing networks from binary data. Sci Rep 4:5918. http://dx.doi.org/10.1038/srep05918.

van Borkulo, C.D., Boschloo, L., Borsboom, D., Penninx, B.W.J.H., Waldorp, L.J., Schoevers, R.A., 2015. Association of symptom network structure with the course of longitudinal depression. JAMA Psychiatry 72:1219-1226. http://dx.doi.org/10.1001/ jamapsychiatry.2015.2079.

van Borkulo, C.D., Borsboom, D., Schroevers, R.A., 2016. Group-level symptom networks in depression-reply. JAMA Psychiatry 73:2016. http://dx.doi.org/10.1001/ jamapsychiatry.2015.3157.

van Borkulo, C.D., Boschloo, L., Kossakowski, J.J., Tio, P., Schoevers, R., Borsboom, D., Lourens, J.W., 2017. Comparing network structures on three aspects: a permutation test. Psychol. Methods http://dx.doi.org/10.13140/RG.2.2.29455.38569.

Bornheimer, L.A., 2016. Moderating effects of positive symptoms of psychosis in suicidal ideation among adults diagnosed with schizophrenia. Schizophr. Res. 176:364-370. http://dx.doi.org/10.1016/j.schres.2016.07.009.

Borsboom, D., 2017. A network theory of mental disorders. World Psychiatry 16:5-13. http://dx.doi.org/10.1002/wps.20375.

Borsboom, D., Cramer, A.O.J., 2013. Network analysis: an integrative approach to the structure of psychopathology. Annu. Rev. Clin. Psychol. 9:91-121. http://dx.doi.org/ 10.1146/annurev-clinpsy-050212-185608

Bos, E.H., Wanders, R.B.K., 2016. Group-level symptom networks in depression (comment). JAMA Psychiatry 73:411-412. http://dx.doi.org/10.1001/ jamapsychiatry.2015.3365.412

Buckley, P.F., Miller, B.J., Lehrer, D.S., Castle, D.J., 2009. Psychiatric comorbidities and schizophrenia. Schizophr. Bull. 35:383-402. http://dx.doi.org/10.1093/schbul/ sbn135.

Chen, J., Chen, Z., 2008. Extended Bayesian information criteria for model selection with large model spaces. Biometrika 95:759-771. http://dx.doi.org/10.1093/biomet/ asn034.

Core Team, R., 2016. R: A Language and Environment for Statistical Computing. R Foundation for Statistical Computing, Vienna, Austria (URL http://www.r-project.org/).

Epskamp, S., Cramer, A.O.J., Waldorp, L.J., Schmittmann, V.D., Borsboom, D., 2012. Qgraph: network visualizations of relationships in psychometric data. J. Stat. Softw. 48:1-18. http://dx.doi.org/10.18637/jss.v048.i04.

Epskamp, S., Borsboom, D., Fried, E.I., 2017. Estimating psychological networks and their accuracy: a tutorial paper. Behav. Res. Methods (Retrieved from https://arxiv.org/ pdf/1604.08462v3). (Doi:10.3758/s13428-017-0862-1)

Foygel, R., Drton, M., 2010. Extended Bayesian information criteria for Gaussian graphical models. Adv. Neural Inf. Proces. Syst. 604-612.

Foygel, R., Drton, M., 2015. Bayesian Model Choice and Information Criteria in Sparse Generalized Linear Models. http://dx.doi.org/10.1038/srep05918.

Fried, E.I., van Borkulo, C.D., Cramer, A.O.J., Boschloo, L., Schoevers, R.A., Borsboom, D., 2016. Mental disorders as networks of problems: a review of recent insights. Soc. Psychiatry Psychiatr. Epidemiol. 52:1-10. http://dx.doi.org/10.1007/s00127-016-1319-z.

Friedman, J., Hastie, T., Tibshirani, R., 2008. Sparse inverse covariance estimation with the graphical lasso. Biostatistics 9:432-441. http://dx.doi.org/10.1093/biostatistics/ kxm045.

Fruchterman, T.M.J., Reingold, E.M., 1991. Graph drawing by force-directed placement. Software-Practice Exp. 21:1129-1164. http://dx.doi.org/10.1002/spe.4380211102.

Golino, H.F., Epskamp, S., 2016. Exploratory Graph Analysis: A new Approach for Estimating the Number of Dimensions in Psychological Research. pp. 1-38

Hill, R.A., 2016. Sex differences in animal models of schizophrenia shed light on the underlying pathophysiology. Neurosci. Biobehav. Rev. 67:41-56. http://dx.doi.org/ 10.1016/j.neubiorev.2015.10.014. 
Isvoranu, A.-M., Borsboom, D., van Os, J., Guloksuz, S., 2016. A network approach to environmental impact in psychotic disorder: brief theoretical framework. Schizophr. Bull. 42:870-873. http://dx.doi.org/10.1093/schbul/sbw049.

Isvoranu, A.-M., van Borkulo, C.D., Boyette, L.-L., Wigman, J.T.W., Vinkers, C.H., Borsboom, D., 2017. A network approach to psychosis: pathways between childhood trauma and psychotic symptoms. Schizophr. Bull. 43:187-196. http://dx.doi.org/10.1093/schbul/ sbw055.

Kay, S.R., Fiszbein, A., Opfer, L.A., 1987. The positive and negative syndrome scale for schizophrenia. Schizophr. Bull. 13:261-276. http://dx.doi.org/10.1093/schbul/ 13.2.261.

Korver-Nieberg, N., Quee, P.J., Boos, H.B., Simons, C.J.P., de Haan, L., Group Investigators, 2012. Genetic risk and outcome of psychosis (GROUP), a multi site longitudinal cohort study focused on gene-environment interaction: objectives, sample characteristics, recruitment and assessment methods. Int. J. Methods Psychiatr. Res. 21:205-221. http://dx.doi.org/10.1002/mpr.

Lako, I.M., Bruggeman, R., Knegtering, H., Wiersma, D., Schoevers, R.A., Slooff, C.J., Taxis, K., 2012. A systematic review of instruments to measure depressive symptoms in patients with schizophrenia. J. Affect. Disord. 140:38-47. http://dx.doi.org/10.1016/ j.jad.2011.10.014.

Leung, A., Chue, P., 2000. Sex differences in schizophrenia, a review of the literature. Acta Psychiatr. Scand. Suppl. 401, 3-38.

Levine, S.Z., Leucht, S., 2016. Identifying a system of predominant negative symptoms: network analysis of three randomized clinical trials. Schizophr. Res. 178:17-22. http://dx.doi.org/10.1016/j.schres.2016.09.002.

Liu, H., Lafferty, J., Wasserman, L., 2009. The nonparanormal: semiparametric estimation of high dimensional undirected graphs. J. Mach. Learn. Res. 10, 2295-2328.

Madhoo, M., Levine, S.Z., 2016. Network analysis of the quick inventory of depressive symptomatology: reanalysis of the STAR*D clinical trial. Eur. Neuropsychopharmacol. 26:1768-1774. http://dx.doi.org/10.1016/j.euroneuro.2016.09.368.
Morgan, V.A., Castle, D.J., Jablensky, A.V., 2008. Do women express and experience psychosis differently from men? Epidemiological evidence from the Australian national study of low prevalence (psychotic) disorders. Aust. N. Z. J. Psychiatry 42:74-82 http://dx.doi.org/10.1080/00048670701732699.

Opsahl, T., Agneessens, F., Skvoretz, J., 2010. Node centrality in weighted networks generalizing degree and shortest paths. Soc. Networks 32, 245-251.

van Rooijen, G., Isvoranu, A.-M., Meijer, C.J., van Borkulo, C.D., Ruhé, H.G., de Haan, L., 2017. A symptom network structure of the psychosis spectrum. Schizophr. Res. http://dx.doi.org/10.1016/j.schres.2017.02.018.

Sarró, S., Dueñas, R.M., Ramírez, N., Arranz, B., Martínez, R., Sánchez, J.M., González, J.M. Saló, L., Miralles, L., San, L., 2004. Cross-cultural adaptation and validation of the Spanish version of the calgary depression scale for schizophrenia. Schizophr. Res. 68: 349-356. http://dx.doi.org/10.1016/S0920-9964(02)00490-5.

Siris, S.G., 2000. Depression in schizophrenia: perspective in the era of "atypical" antipsychotic agents. Am. J. Psychiatry 157:1379-1389. http://dx.doi.org/10.1176 appi.ajp.157.9.1379.

Tibshirani, R., 1996. Regression shrinkage and selection via the lasso. J. R. Stat. Soc. 58 , 267-288.

Wichers, M., Wigman, J.T.W., Bringmann, L.F., de Jonge, P., 2017. Mental disorders as networks: some cautionary reflections on a promising approach. Soc. Psychiatry Psychiatr. Epidemiol. 52:143-145. http://dx.doi.org/10.1007/s00127-016-1335-z.

Wigman, J.T.W., Os van, J., Borsboom, D., Wardenaar, K.J., Epskamp, S., Klippel, A., Merge W., Viechtbauer, Myin-Germeys I., Wichers, M., 2015. Exploring the underlying structure of mental disorders: cross-diagnostic differences and similarities from a network perspective using both a top-down and a bottum-up approach. Psychol. Med. 45, 2375-2387. 